\title{
Separating propagating and non-propagating dynamics in fluid-flow equations
}

Samuel Sinayoko,

A. Agarwal and Z. Hu

University of Southampton

Institute of Sound and Vibration Research

\section{May 2009}




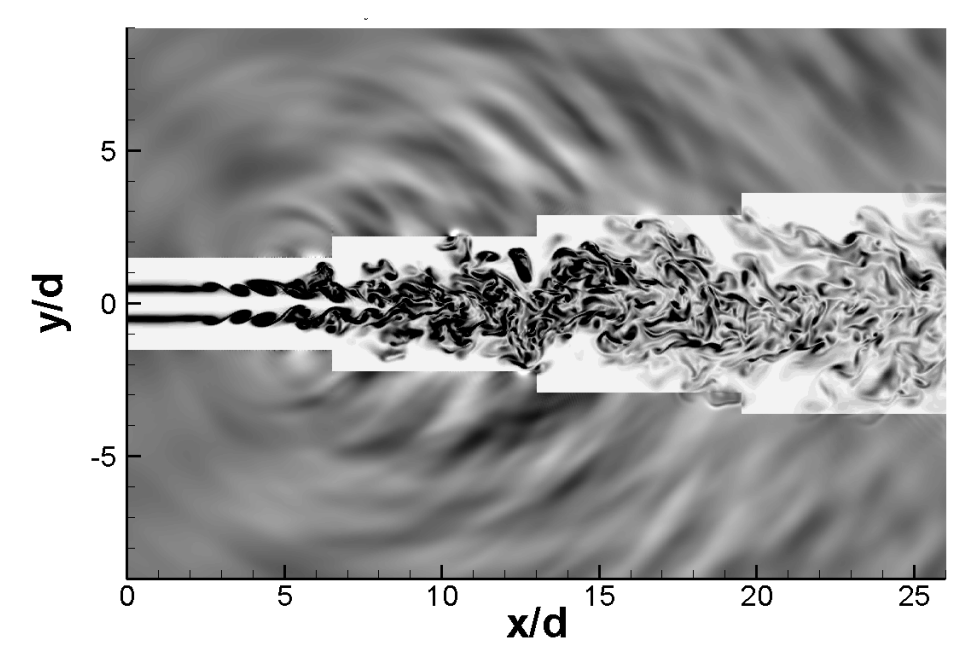

S. Sinayoko, A. Agarwal, Z. Hu

Sep. propagating \& non-propagating dynamics in fluid-flow 


\section{Introduction}

How to define the physical sources of sound?

Objectives

(1) Derive an expression for the physical sources of sound.

(2) Demonstrate that it is possible to separate the radiating and non-radiating parts of the flow.

(3) Compute the physical sources of sound. 


\section{Goldstein's theory}

Equations

\section{Goldstein's theory}

\section{Jet}

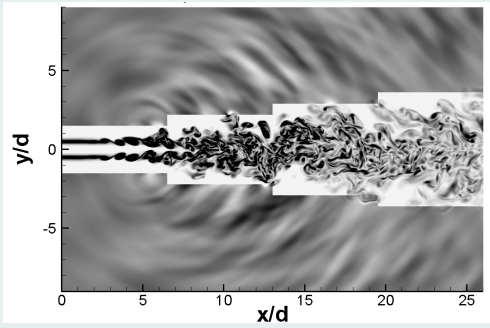

S. Sinayoko, A. Agarwal, Z. Hu

\section{Filtered jet}

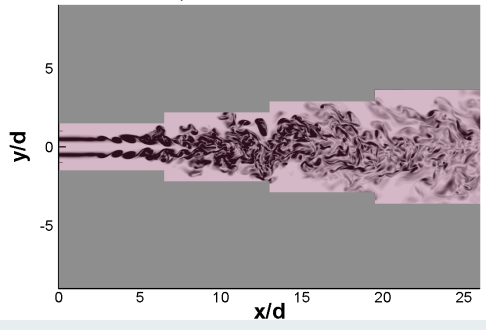




\section{Goldstein's theory}

Equations

\section{Goldstein's theory}

Jet

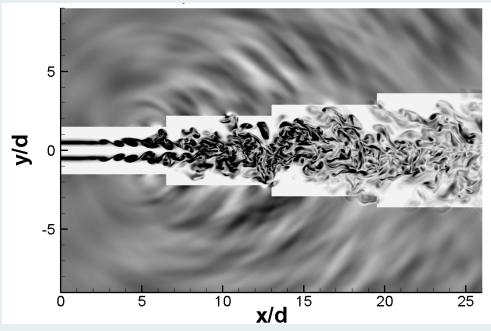

\section{Filtered jet}

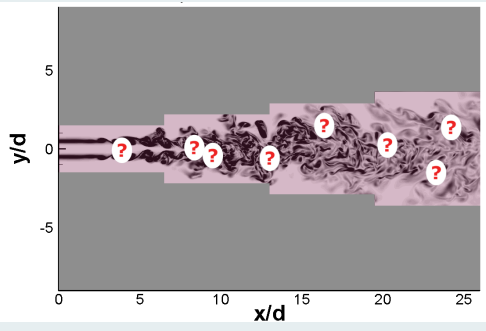




\section{Goldstein's theory}

Jet

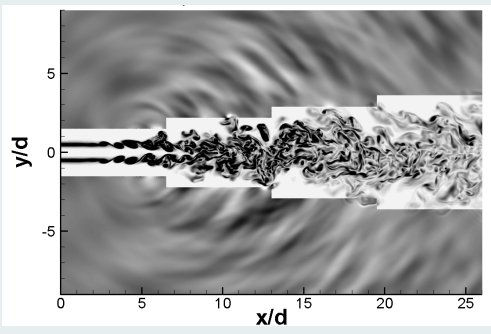

These sources should be close to the true sources of sound.
Filtered jet

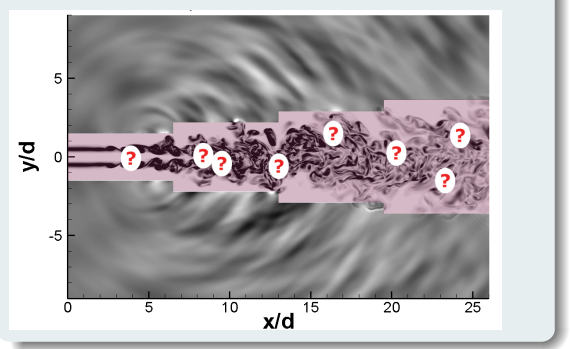




\section{Governing equation for fluctuating quantities}

Flow filtering

$$
\mathcal{L} f=\bar{f}
$$

Flow decomposition

$$
f=\bar{f}+f^{\prime}
$$

Conservation of mass

$$
\begin{aligned}
& \frac{\partial \rho}{\partial t}+\frac{\partial \rho v_{j}}{\partial x_{j}}=0 \\
& \frac{\partial \bar{\rho}}{\partial t}+\frac{\partial \overline{\rho v_{j}}}{\partial x_{j}}=0 .
\end{aligned}
$$

Conservation of mass for fluctuating quantities

$$
\frac{\partial \rho^{\prime}}{\partial t}+\frac{\partial\left(\rho v_{j}\right)^{\prime}}{\partial x_{j}}=0
$$




\section{Governing equation for fluctuating quantities}

Conservation of mass for fluctuating quantities

$$
\frac{\partial \rho^{\prime}}{\partial t}+\frac{\partial\left(\rho v_{j}\right)^{\prime}}{\partial x_{j}}=0
$$

Momentum conservation for fluctuating quantities

$$
\frac{\partial\left(\rho v_{i}\right)^{\prime}}{\partial t}+\frac{\partial\left(\rho v_{i} v_{j}\right)^{\prime}}{\partial x_{j}}+\frac{\partial p^{\prime}}{\partial x_{i}}=\frac{\partial \sigma_{i j}^{\prime}}{\partial x_{j}}
$$

Taking $\partial(6) / \partial x_{i}-\partial(5) / \partial t$ gives

$$
\frac{\partial^{2} p^{\prime}}{\partial x_{i} x_{i}}-\frac{\partial^{2} \rho^{\prime}}{\partial t^{2}}+\frac{\partial^{2}\left(\rho v_{i} v_{j}\right)^{\prime}}{\partial x_{i} \partial x_{j}}=\frac{\partial^{2} \sigma_{i j}^{\prime}}{\partial x_{i} \partial x_{j}} .
$$




\section{Governing equation for fluctuating quantities}

Favre averaging, $\tilde{f}=\overline{\rho f} / \bar{\rho}$,

Governing equation

$$
\frac{\partial^{2} p^{\prime}}{\partial x_{i} \partial x_{i}}-\frac{\partial^{2} \rho^{\prime}}{\partial t^{2}}+\frac{\partial^{2}}{\partial x_{i} \partial x_{j}}\left(\tilde{v}_{i} \tilde{v}_{j} \rho^{\prime}+\bar{\rho} \tilde{v}_{j} v_{i}^{\prime}+\bar{\rho} \tilde{v}_{i} v_{j}^{\prime}\right)=\frac{\partial^{2} \sigma_{i j^{\prime}}}{\partial x_{i} \partial x_{j}}+s
$$

Source definition

$$
\begin{aligned}
s & =-\frac{\partial^{2}}{\partial x_{i} \partial x_{j}}\left(T_{i j}+\rho v_{i}^{\prime} v_{j}^{\prime}+\tilde{v}_{i} \rho^{\prime} v_{j}^{\prime}+\tilde{v}_{j} \rho^{\prime} v_{i}^{\prime}\right) \\
T_{i j} & =-\bar{\rho}\left(\widetilde{v_{i} v_{j}}-\tilde{v}_{i} \tilde{v}_{j}\right) .
\end{aligned}
$$




\section{SouthlunversTY of}

Institute of Sound and

Vibration Research

Filter defining properties

Local filter

Global filter

\section{Problem description}

\section{Parallel flow}

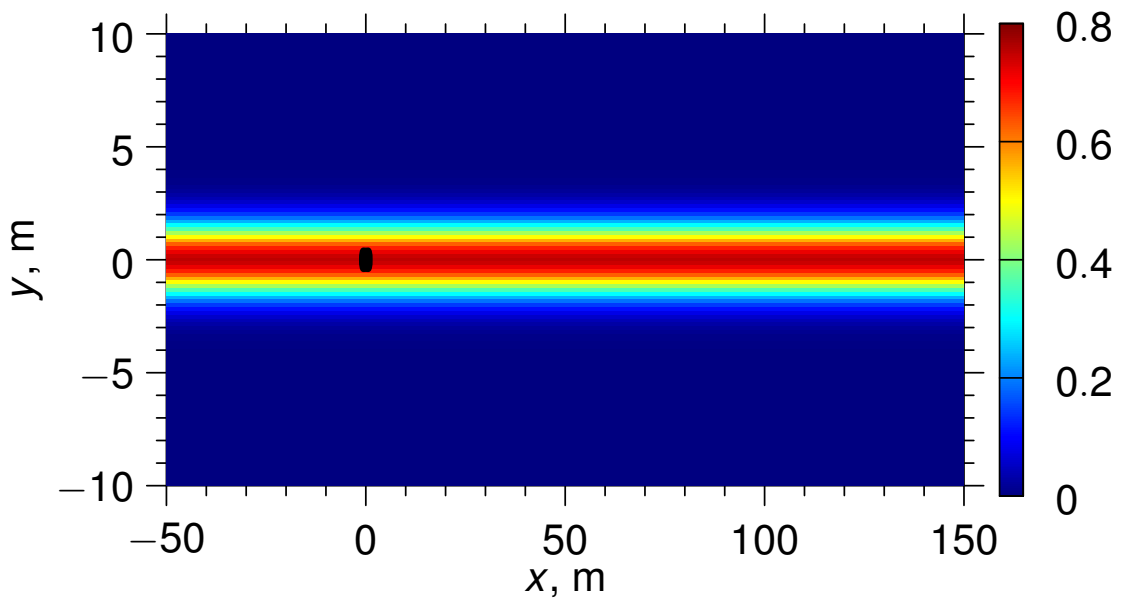




\section{Problem description}

Filter defining properties

Local filter

Global filter

\section{Problem description}

\section{Pressure field}

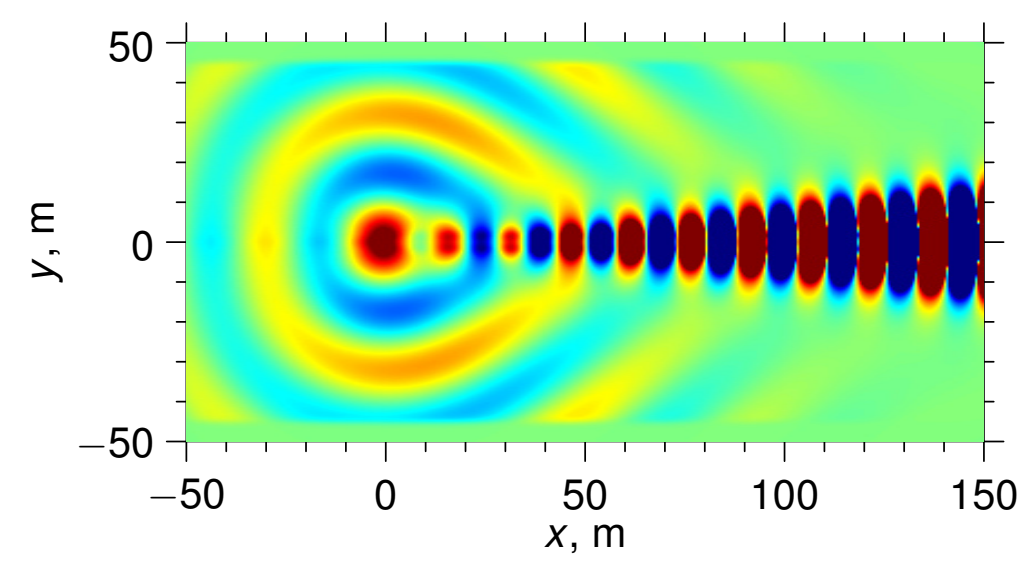




\section{Defining properties}

Fourier transform

$$
\begin{aligned}
f(\mathbf{x}, t) & \rightarrow F(\mathbf{k}, \omega) \\
\bar{f}(\mathbf{x}, t) & \rightarrow \bar{F}(\mathbf{k}, \omega)
\end{aligned}
$$

Non-radiating condition

$$
\bar{F}(\mathbf{k}, \omega)=0 \quad \text { for } \quad|\mathbf{k}|=\frac{|\omega|}{C_{\infty}}
$$

Additional requirement

$$
\bar{F}(\mathbf{k}, \omega)=F(\mathbf{k}, \omega) \quad \text { for } \quad|\mathbf{k}| \neq \frac{|\omega|}{c_{\infty}}
$$




\section{Local filter}

\section{Filter definition}

D’Alembertian filter

$$
\bar{f}(\mathbf{x}, t)=\left(\frac{1}{c_{\infty}^{2}} \frac{\partial^{2}}{\partial t^{2}}-\nabla^{2}\right) f(\mathbf{x}, t),
$$

Frequency domain

$$
\bar{F}(\mathbf{k}, \omega)=\left(|\mathbf{k}|^{2}-\frac{\omega^{2}}{c_{\infty}^{2}}\right) F(\mathbf{k}, \omega)
$$

$$
\Rightarrow \bar{F}(\mathbf{k}, \omega)=0 \quad \text { for } \quad|\mathbf{k}|=\frac{|\omega|}{c_{\infty}}
$$


Problem description

Filter defining properties

Local filter

Global filter

\section{Local filter}

\section{Results}

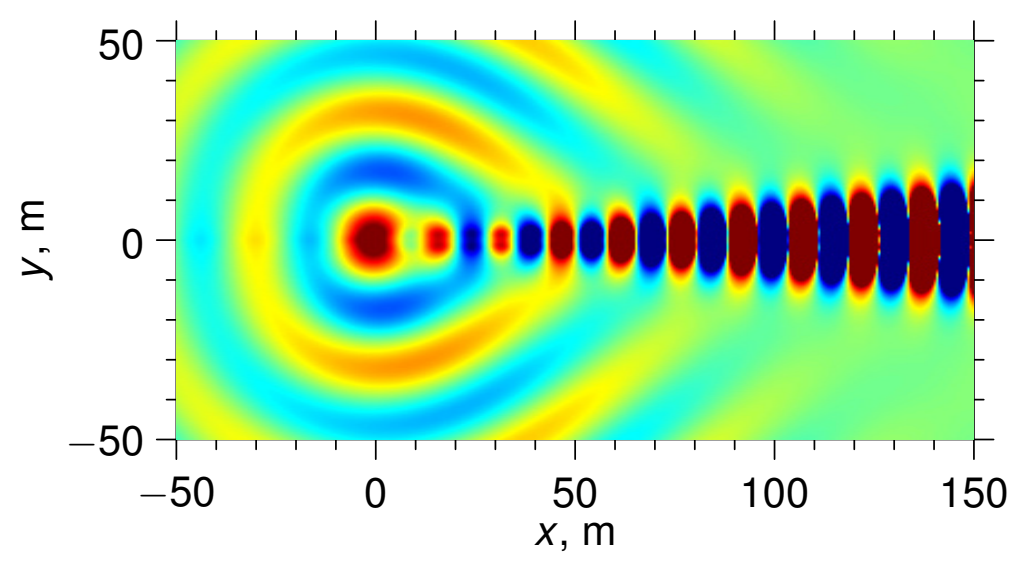

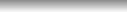




\section{Local filter}

\section{Results}

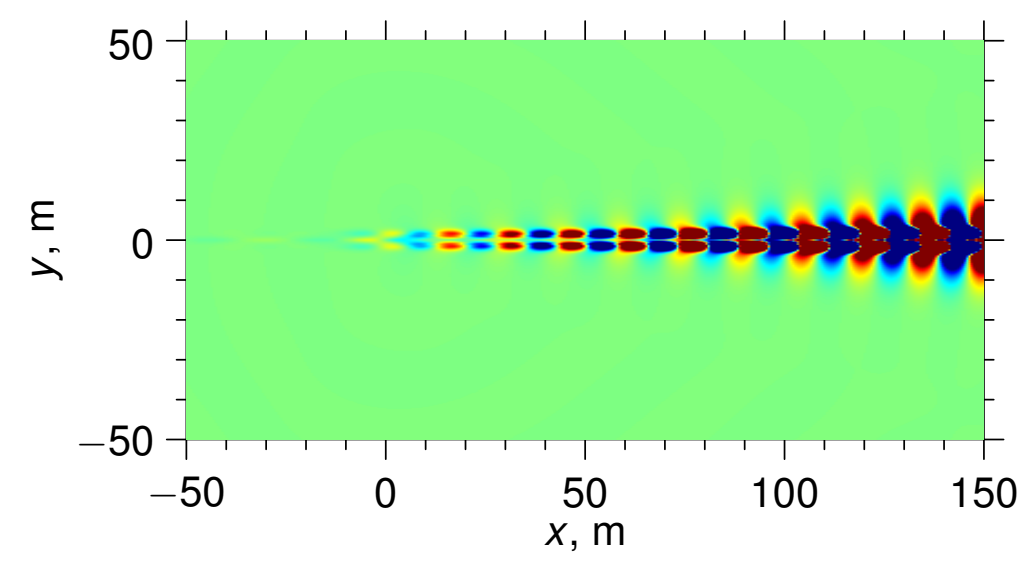
(n) 


\section{Global filter}

\section{Filter definition}

Time-domain

$$
\bar{f}=w * f
$$

Frequency-domain

$$
\bar{F}=W F
$$

$$
f(\mathbf{x}, t) \stackrel{\text { F.T. }}{\longrightarrow} F(\mathbf{k}, \omega)
$$

Filter window

$$
\begin{cases}0 & \text { if }|\mathbf{k}|=|\omega| / c_{\infty} \\ 1 & \text { otherwise }\end{cases}
$$

$$
\bar{f}(\mathbf{x}, t) \stackrel{\text { I.F.T. }}{\longleftarrow} \bar{F}(\mathbf{k}, \omega)
$$

S. Sinayoko, A. Agarwal, Z. Hu 


\section{Global filter}

\section{Filter definition}

\section{Gaussian filter}

$$
W(\mathbf{k}, \omega)=\exp \left(-\frac{\left(k_{x}-\alpha\right)^{2}}{2 \sigma^{2}}\right)+\exp \left(-\frac{\left(k_{x}+\alpha\right)^{2}}{2 \sigma^{2}}\right)
$$

$$
\alpha=0.68 \mathrm{~m}^{-1}, \quad \sigma=0.1 \mathrm{~m}^{-1} .
$$

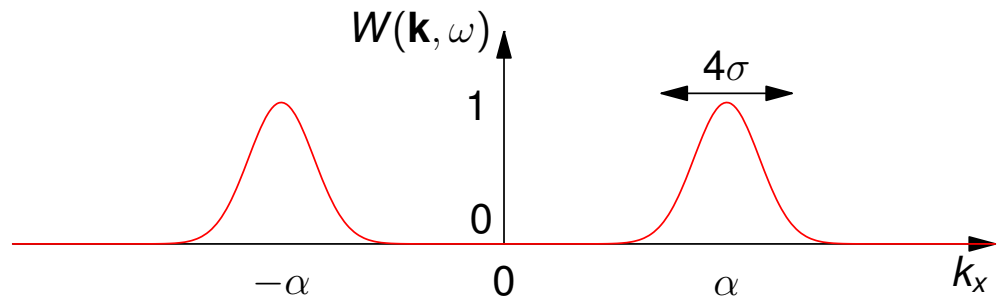


Problem description

Filter defining properties

Local filter

Global filter \\ Global filter}

\section{Results}

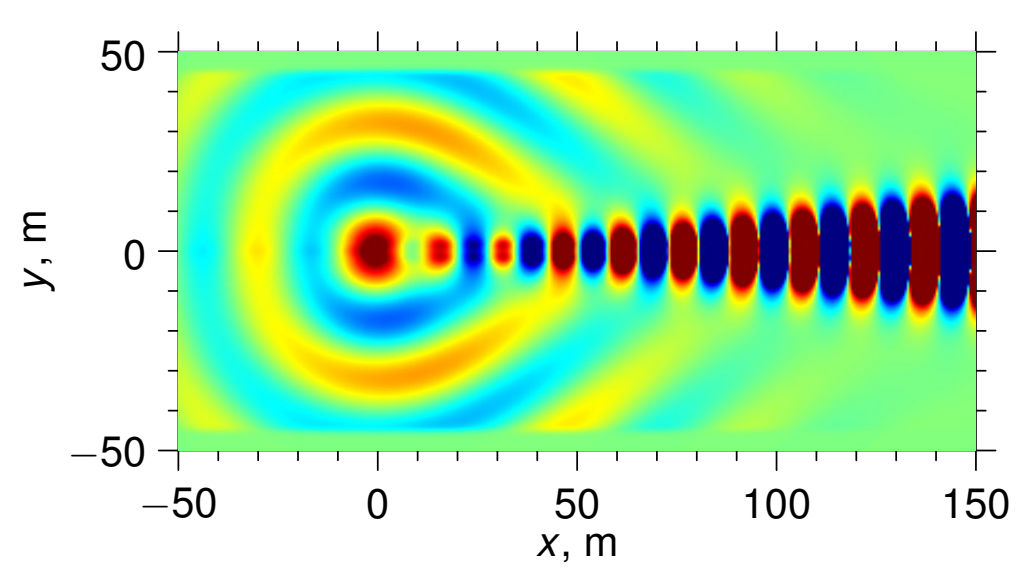

Conclusion 
Problem description

Filter defining properties

Local filter

Global filter

\section{Global filter}

\section{Results}

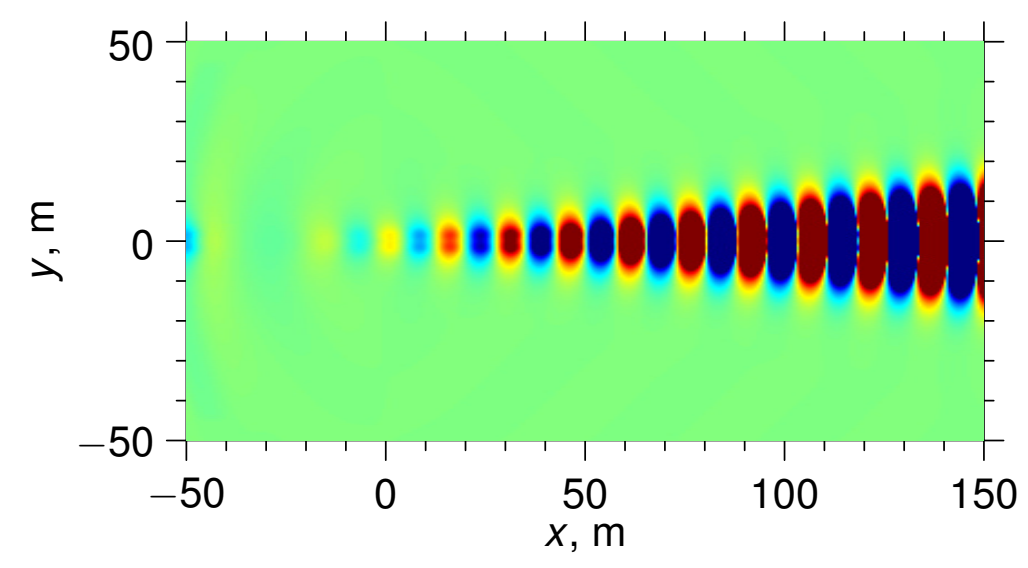

\section{Global filter} (a) 


\section{Global filter}

\section{Validation}

Comparison with analytical result along profile $y=15 \mathrm{~m}$

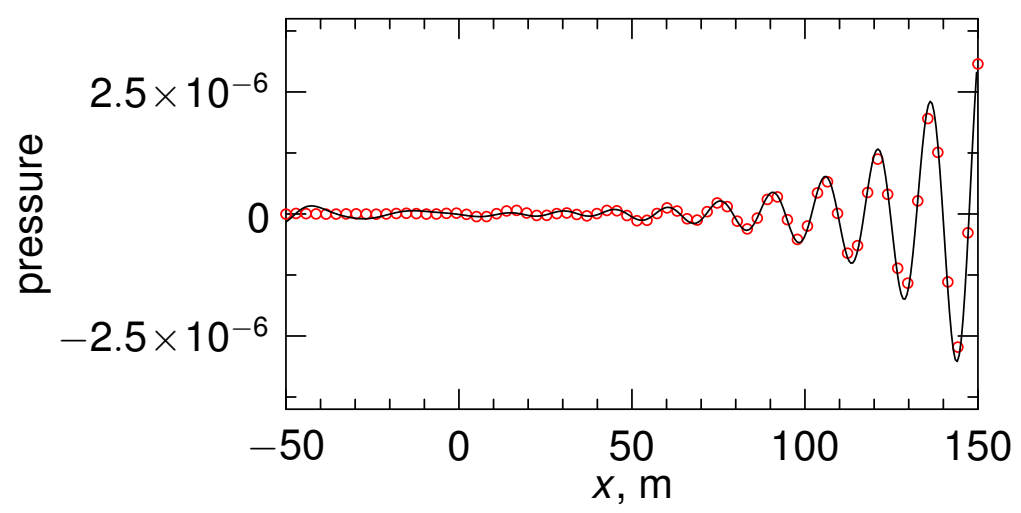




\section{Flow description}

\section{Mean flow}

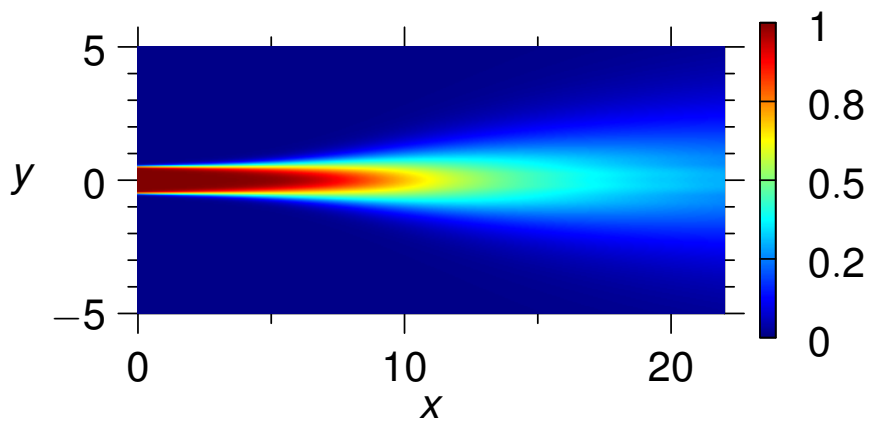

Mean flow excited at two frequencies:

$$
\omega_{1}=2.2, \quad \omega_{2}=3.4, \quad \Delta \omega=1.2 \text {. }
$$




\section{Flow description}

\section{Pressure field

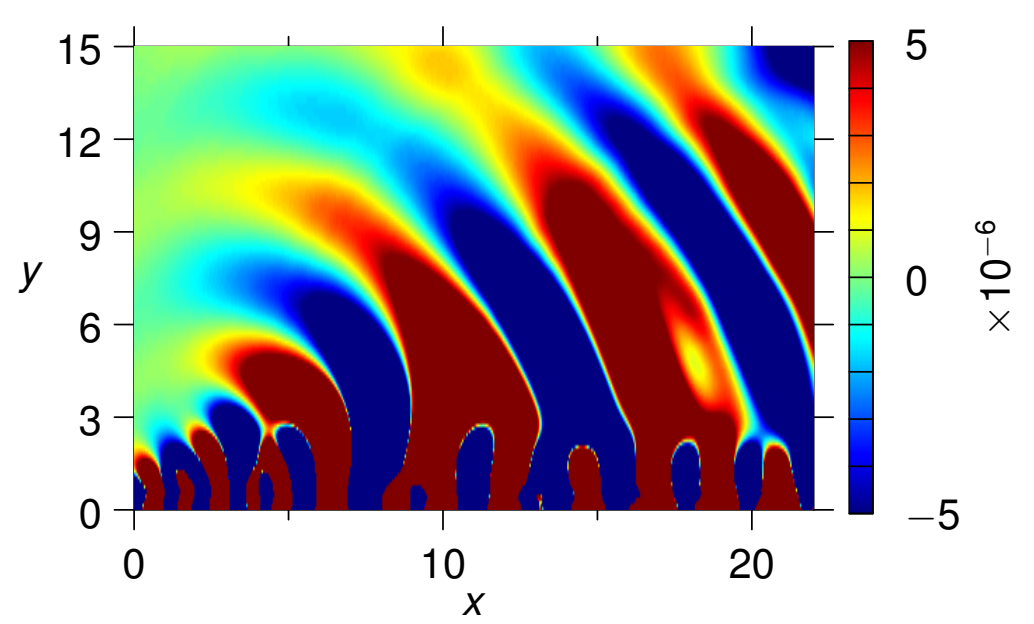




\section{Flow description}

\section{Frequency analysis}

Hydrodynamic region

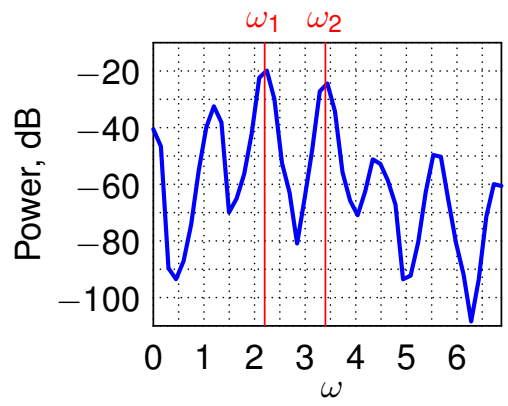

\section{Acoustic region}

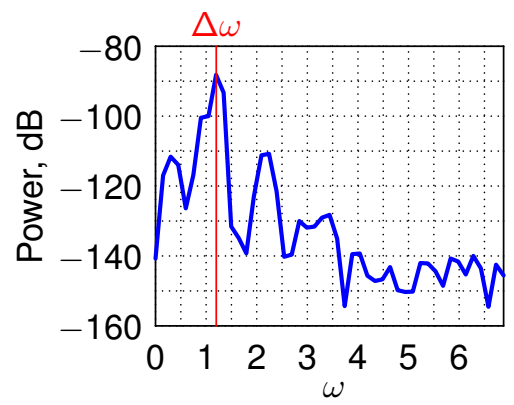




\section{Flow description}

\section{Frequency analysis}

Hydrodynamic region

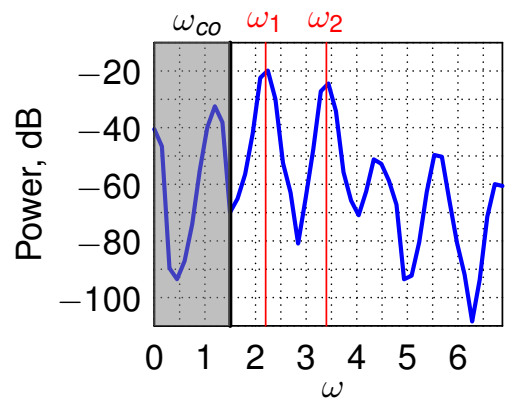

\section{Acoustic region}

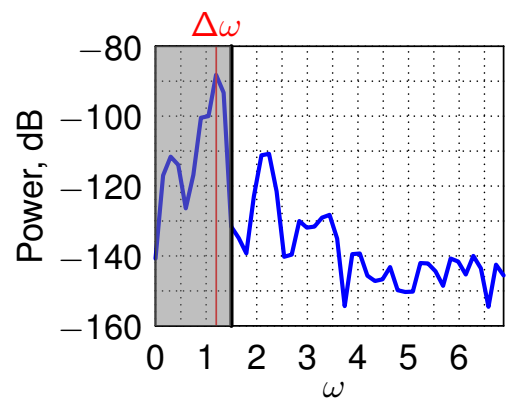




\section{Filter design}

\section{Definition}

\section{Tanh filter}

$$
W(\mathbf{k}, \omega)=\frac{1}{2}\left[1+\tanh \left(\frac{|\mathbf{k}|-k_{c o}}{\sigma}\right)\right],
$$

$$
k_{c o}=1.3, \quad \sigma=0.2 .
$$
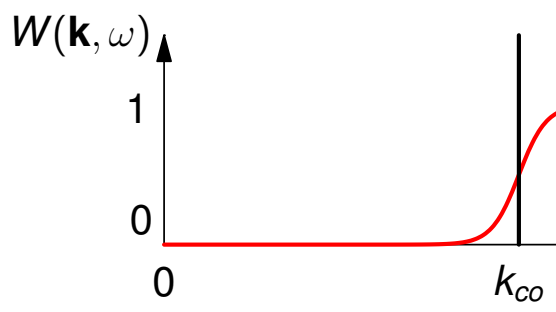

$\mid \mathbf{k}$ 


\section{Southampton Institute of Sound and}

Vibration Research

\section{Flow description}

Filter design

Sound sources

\section{Filter design}

\section{Validation}

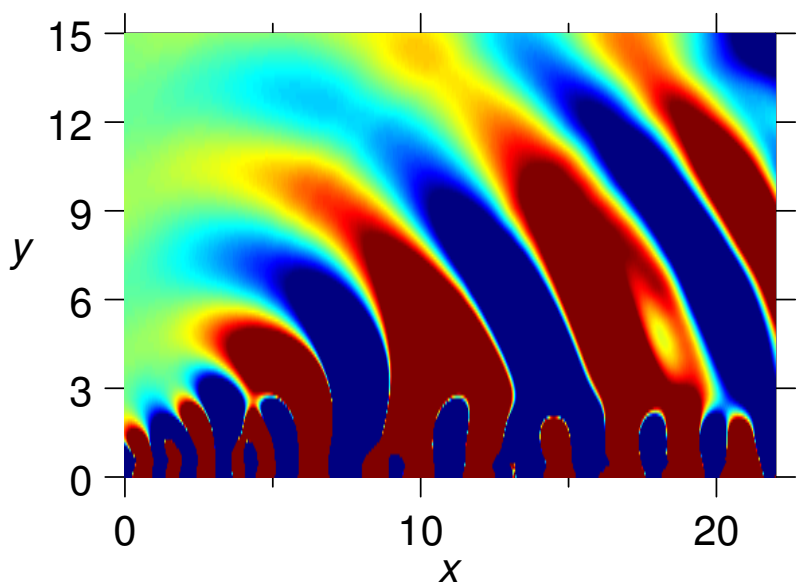

\section{Pressure field $p$}




\section{Southampton Institute of Sound and}

Vibration Research

Flow description

Filter design

Sound sources

\section{Filter design}

\section{Validation}

Filtered pressure $\bar{p}$

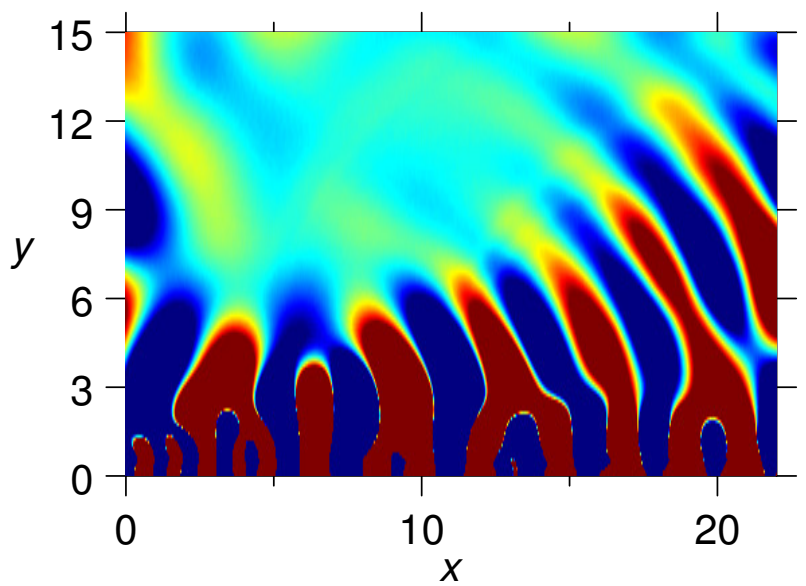


Defining the physical sources of sound

Non-radiating filter design

Sources of sound in an axi-symmetric jet
Flow description

Filter design

Sound sources

\section{Filter design}

\section{Validation}

Fluctuating pressure $p^{\prime}$

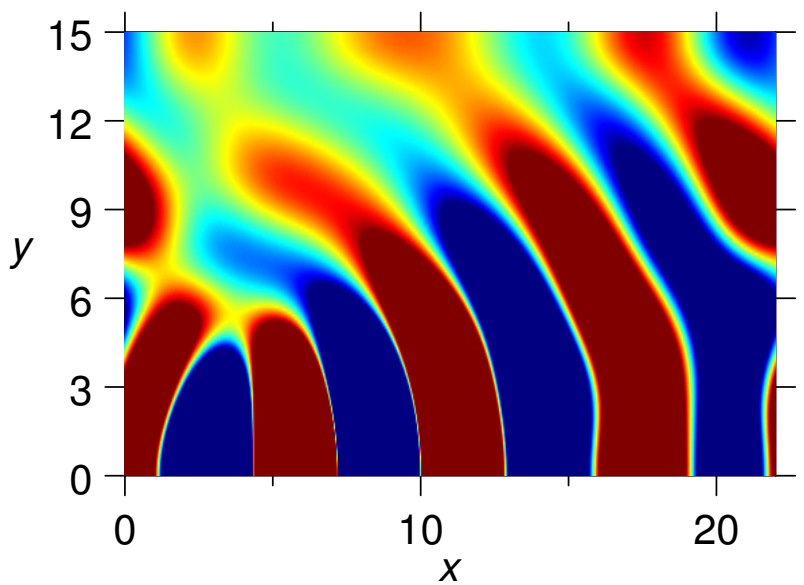




\section{Sound sources}

\section{Using non-radiating filter}

Sound source $s$

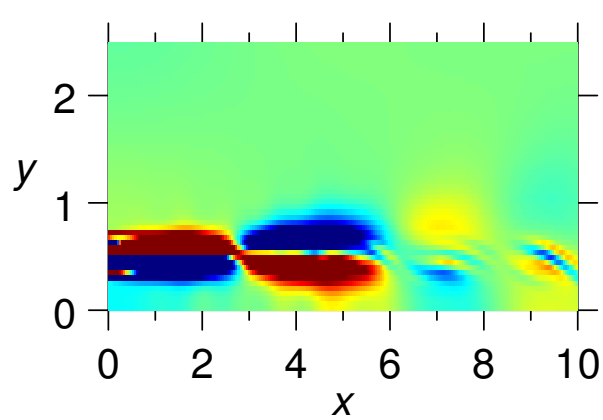

Spectrum at $(4.0,0.55)$

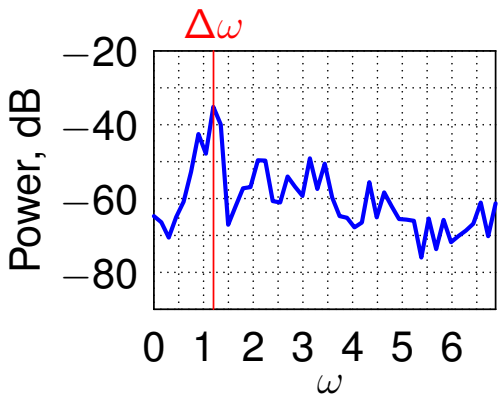




\section{Sound sources}

\section{Using time average filter}

Sound source $s$

\section{Spectrum at $(5.5,0.5)$}
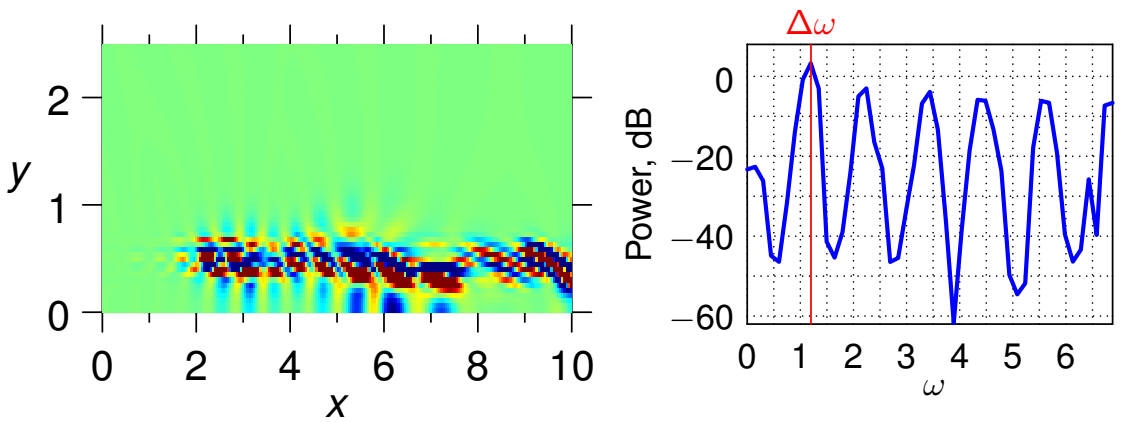


\section{Sound sources}

\section{Evolution in time}

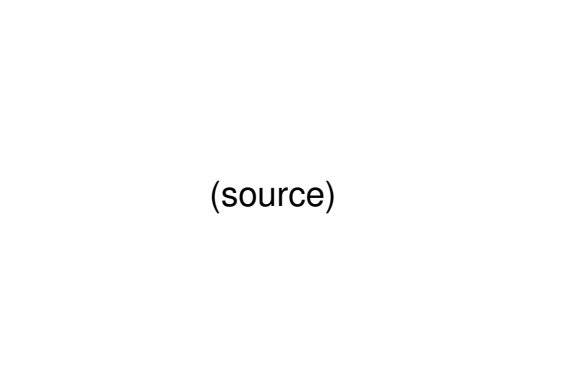




\section{Conclusion and future work}

Results

- Sound source definition

- Separation possible with convolution filters.

- Clearer physical interpretation of the sources.

Future work

- Mixing-layer and a two-dimensional jet.

- Physical mechanism behind the sound sources. 


\section{Acknowledgements}

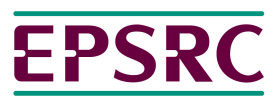

Engineering and Physical Sciences Research Council
Rolls-Royce 